CAS-KITPC/ITP-090

\title{
Multi-Stream Inflation
}

\author{
Miao Li* and Yi Wangt \\ Kavli Institute for Theoretical Physics China, \\ Key Laboratory of Frontiers in Theoretical Physics, Institute of Theoretical Physics, \\ Chinese Academy of Sciences, Beijing 100190, P.R.China
}

\begin{abstract}
We propose a "multi-stream" inflation model, which is a double field model with spontaneous breaking and restoration of an approximate symmetry. We calculate the density perturbation and non-Gaussianity in this model. We find that this model can have large, scale dependent, and probably oscillating non-Gaussianity. We also note that our model can produce features in the CMB power spectrum and hemispherical power asymmetry.
\end{abstract}

*Electronic address: mli@itp.ac.cn

${ }^{\dagger}$ Electronic address: wangyi@itp.ac.cn 


\section{INTRODUCTION}

Inflation has become one of the most important windows to connect cosmological observations to fundamental physics, such as string theory. Recent studies on string theory show evidence that inflation may happen in the string theory landscape [1] with extremely complicated inflaton potential [2].

One lesson from the string landscape is that the inflationary dynamics may be not as simple as one expected before, and more possibilities during inflation should be taken into consideration. Inflation in the string landscape opens up a number of possibilities to test string theory by the stringy imprints on the CMB temperature fluctuations.

On the other hand, in the recent analysis of CMB anisotropy, there are several hints for new physics. If future experiments confirm some of these possibilities, they will pose challenges for inflationary cosmology and opportunities for string theory. These challenges and opportunities include:

- Non-Gaussianity. The WMAP5 bound for local non-Gaussianity is [3]

$$
-9<f_{N L}<111(95 \% \mathrm{CL})
$$

The result is still consistent with $f_{N L} \simeq 0$. However, the central value of $f_{N L}$ is large and positive. If integration of more years of WMAP or the Planck mission confirm this result, it would rule out the simplest inflation model. The theoretical investigation of non-Gaussianity include [4, 5, 6, 7, 8, 9, 10, 11, 12, 13, 14, 15, 16, 17]. As there is a consistency relation [13, 18] for single field inflation, a large and local non-Gaussianity shows evidence that inflation should involve more than one field.

- Features in the CMB. As shown in [19], there may be features in the CMB scalar power spectrum. A feature in the CMB spectrum implies a departure from the standard single field slow roll inflation at a certain scale.

- Hemispherical power asymmetry. As shown in [20], the temperature-fluctuation amplitude is larger by roughly $10 \%$ in one hemisphere of the CMB map than in the other. Again, this asymmetry can not be explained by the simplest inflation model. It would also be interesting to see whether this difference of power also happens on smaller scales in the sky. 
In this paper, we propose a "multi-stream" inflation model, which is a double field inflation model with spontaneous breaking and restoration of an approximate symmetry. We find that with appropriate choice of parameters, our model is consistent with current observations, and can produce large non-Gaussianity, features in the CMB and hemispherical power asymmetry. In Section 2, we describe the picture of multi-stream inflation and calculate the density perturbations. In Section 3, we calculate the non-Gaussianity of multi-stream inflation. In Section 4, we give a summary of the signatures for multi-stream inflation in different parameter regions. We conclude in Section 5.

\section{MULTI-STREAM INFLATION}

In this section, we investigate the background evolution and the scalar perturbation spectrum of multi-stream inflation. We consider a period of inflation involving two fields.

We consider the potential $V(\varphi, \chi)$ as illustrated in Fig.1. In the beginning, the $\varphi$ direction

is the inflationary direction. As $\varphi$ evolves to $\varphi_{1}$, the $\chi$ direction becomes a tachyonic direction. At this point, the inflationary trajectory spontaneously breaks into two paths, namely $A$ and $B$ in Fig,1. The whole picture looks like a stream which splits and flows along both sides of a hill. So we call this scenario "multi-stream" inflation.

As inflation continues, depending on the potential and the reheating time, the trajectories

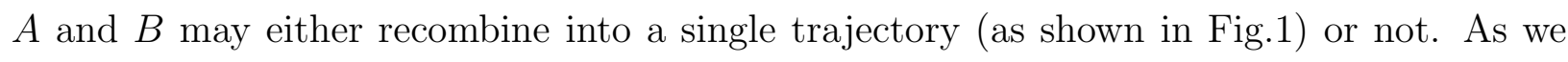
will see in the calculation of scalar perturbations, if $A$ and $B$ do not recombine and reheat separately, one typically has large anisotropy at the symmetry breaking scale, and requires more fine tuning to fit the CMB power spectrum. So in this note, we mainly discuss the former possibility.

Now let us investigate the perturbations. The perturbations of multi-stream inflation should be the same as in usual inflation models except in the regime between $\varphi_{1}$ and $\varphi_{2}$. Moreover, if the potential energy of path $A$ and path $B$ are identical, multi-stream inflation also does not differ much from usual inflation models except for some amount of isocurvature perturbations, because in this case, to choose which way is not important.

To have more interesting physics, we assume the paths $A$ and $B$ have a slightly different potential energy $\delta V \equiv V\left(\varphi, \chi_{A}\right)-V\left(\varphi, \chi_{B}\right)$, with $\delta V$ non-vanishing only if $\varphi_{1}<\varphi<\varphi_{2}$. After this setup, multi-stream inflation will have several interesting differences from the 


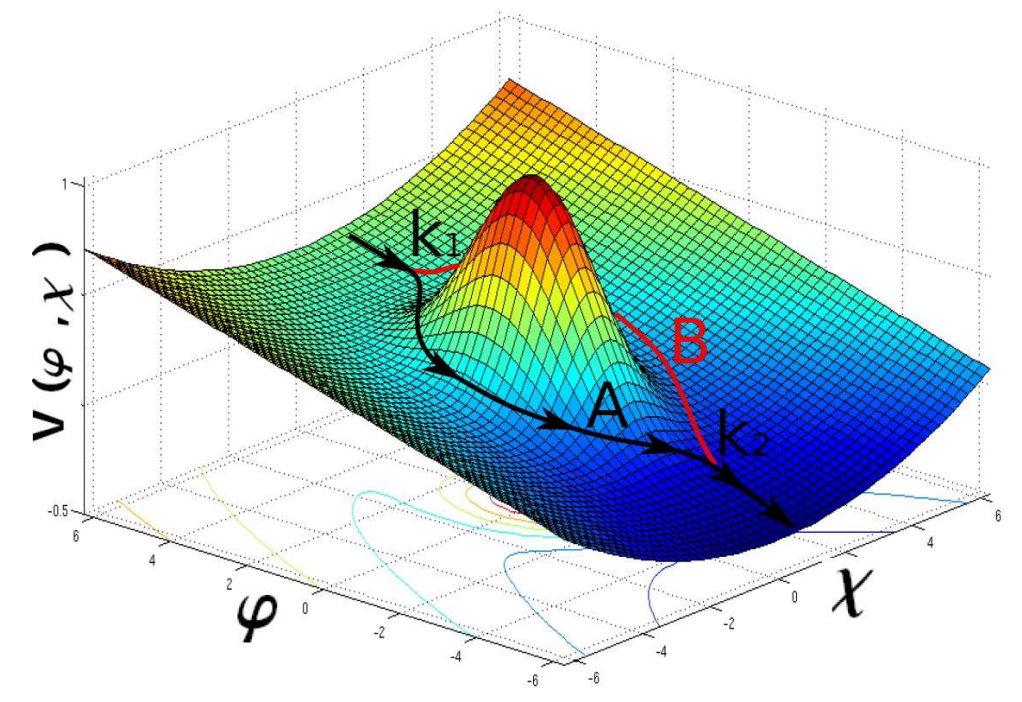

FIG. 1: In this figure, we illustrate the picture of multi-stream inflation. As the inflaton rolls down the potential, it experiences a spontaneously breaking of approximate symmetry at $k_{1}$, with $\varphi=\varphi_{1}$. After that, the inflation trajectory branches off into two paths denoted by $A$ and $B$. From the viewpoint of the CMB map, some parts of the sky with size of order $1 / k_{1}$ come from path $A$, and other parts of the sky come from $B$. At $k_{2}$, with $\varphi=\varphi_{2}$, the approximate symmetry is restored and the inflationary direction becomes unique again.

usual inflation model.

Firstly, the perturbations along trajectories $A$ and $B$ have different power spectra. It is because the tilt of the potential is typically different when $\delta V \neq 0$. To be more explicit, the power spectra along the two trajectories are calculated using the local Hubble parameter and the local slow roll parameters as

$$
P_{\zeta}^{A}=\left.\frac{H^{2}}{8 \pi^{2} M_{p}^{2} \epsilon}\right|_{A} \quad P_{\zeta}^{B}=\left.\frac{H^{2}}{8 \pi^{2} M_{p}^{2} \epsilon}\right|_{B} .
$$

The difference in the power spectra satisfies

$$
\left(P_{\zeta}^{A}-P_{\zeta}^{B}\right) / P_{\zeta}^{A} \sim \delta V / V
$$

In position space, trajectories $A$ and $B$ correspond to different parts of the sky. So a difference between the power spectra $P_{\zeta}^{A}$ and $P_{\zeta}^{B}$ implies that on cosmological scales corresponding to $\varphi_{1}$ and $\varphi_{2}$, the perturbations in some regions of the sky have more power, and in some other regions of the sky have less power. 
For example, if $k_{1}$ corresponds to the scale of the whole observable universe, then trajectories $A$ and $B$ both span about half of the sky. In this case, multi-stream inflation provides a possible solution to the hemispherical power asymmetry problem [20].

If the hemispherical power asymmetry is really there and with a difference of order $10 \%$ in magnitude, we need $\delta V / V \sim 10^{-2}$, and $k_{1}$ to be the scale that comparable to the present Hubble scale to explain it. As shown in the following sections, in this case, the corresponding non-Gaussianity is typically $f_{N L} \sim \mathcal{O}(100)$.

In multi-stream inflation, $k_{1}$ could also correspond to much smaller length scales compared with the present Hubble scale, depending on the explicit form of the inflationary potential. In this case, the multi-stream effect generates about $\left(k_{1} / k_{0}\right)^{2}$ regions on the $\mathrm{CMB}$, where $k_{0}$ is the comoving wave number whose wavelength is the whole observable universe. In these regions, the primordial power spectrum either takes the form $P_{\zeta}^{A}$ or $P_{\zeta}^{B}$.

Secondly, there can be a difference of e-folding numbers for paths $A$ and $B$. This difference results in a time delay effect for the reheating surface, thus producing extra perturbation.

To be explicit, let $\delta N \equiv N_{A}-N_{B}$, where $N_{A}$ and $N_{B}$ are the background e-folding numbers evaluated along trajectories $A$ and $B$ respectively. We note that $\delta N$ depends on the detailed form of the potential

$$
\delta N=\frac{1}{M_{p}^{2}} \int_{\varphi_{1}}^{\varphi_{2}} d \varphi \frac{V+\delta V}{V^{\prime}+\delta V^{\prime}}-\frac{1}{M_{p}^{2}} \int_{\varphi_{1}}^{\varphi_{2}} d \varphi \frac{V}{V^{\prime}},
$$

where a prime denotes a derivative with respect to $\varphi$. We denote the comoving scale that exits the Hubble radius when $\varphi=\varphi_{1}$ by $k_{1}$, and $\varphi=\varphi_{2}$ by $k_{2}$ as shown in Fig,1. $\delta N$ provides an extra source of curvature perturbation on the scale $k_{1}$. It is because the existence of $\delta N$, thus the time delay in reheating divides the CMB sky into patches with comoving length scale $1 / k_{1}$. This anisotropy corresponds to a perturbation $\delta \zeta_{k_{1}}^{N}$. The amount of perturbation can be calculated as [21]

$$
\zeta_{k_{1}}=\Delta N=\frac{H}{\dot{\varphi}} \delta \varphi+\delta N \equiv \zeta_{0}+\delta \zeta_{k_{1}}^{N},
$$

where $\Delta N$ is the total e-folding number difference between path $A$ and path $B$. The difference $\Delta N$ has two sources, namely the quantum fluctuation of the inflation $\delta \varphi$, and the intrinsic difference in the e-folding number $\delta N$ between the two paths.

The total power spectrum takes the form

$$
P_{\zeta_{k_{1}}} \sim \zeta_{0}^{2}+\left(\delta \zeta_{k_{1}}^{N}\right)^{2}
$$


We neglected the cross term $\zeta_{0} \delta \zeta_{k_{1}}^{N}$, because if one does not consider the conversion from isocurvature perturbation to curvature perturbation, then $\zeta_{0}$ and $\delta \zeta_{k_{1}}^{N}$ are uncorrelated. We will return to this issue and give a more careful analysis of Eq. (66) in the next section.

The existence of $\delta N$ is a mixed blessing. The good news is that $\delta N$ provides a nice explanation for the features in the CMB power spectrum, because the jump of $\zeta$ takes place only in a very sharp range of $k$. The bad news is that when $\delta V$ is not very small, to make this jump not too large, one needs some amount of tuning to be consistent with experiments. We shall estimate the amount of tuning in Section 4.

\section{NON-GAUSSIANITY OF MULTI-STREAM INFLATION}

In this section, we calculate the non-Gaussianity of multi-stream inflation. We shall neglect the non-Gaussianity produced by mechanisms other than the multi-stream effect.

To perform the calculation, we further assume that the mass of $\chi$ shortly before the Hubble exit of $k_{1}$ is of order $H$. This assumption guarantees that $m_{\chi}$ is not too light to randomly walk more than one e-fold away from the origin, and also guarantees that $m_{\chi}$ is not too heavy to have an effect.

As the inflation trajectory turns at $\varphi_{1}$, a certain amount of isocurvature perturbation $\delta \chi_{k_{1}}$ is projected to curvature perturbation. Note that the sign of $\delta \chi_{k_{1}}$ decides whether inflation will be along trajectory $A$ or $B$. The observed curvature perturbation at $k_{1}$ also includes a contribution from $\delta N$, we have

$$
\zeta_{k_{1}} \equiv \zeta_{0}+\delta \zeta_{k_{1}}, \quad \delta \zeta_{k_{1}} \equiv c \frac{H}{\dot{\varphi}} \delta \chi_{k_{1}}+\delta N \equiv \delta \zeta_{k_{1}}^{S}+\delta \zeta_{k_{1}}^{N}
$$

where $c$ denotes the fraction of isocurvation which is projected onto the curvature perturbation during the change of the trajectory, $\delta \zeta_{k_{1}}^{S}$ and $\delta \zeta_{k_{1}}^{N}$ denote the curvature perturbation from projection of isocurvature perturbation and from $\delta N$ respectively.

There are two kinds of non-Gaussianities in multi-stream inflation. Firstly, the one-point probability distribution function for $\zeta_{k_{1}}$ is non-Gaussian. This is because the extra density fluctuation $\delta \zeta_{k_{1}}^{N}$, coming from the e-folding number difference between trajectories $A$ and $B$, peaks at two particular values:

$$
P\left(\delta \zeta_{k_{1}}^{N}\right)=\frac{1}{2}\left[\delta\left(\delta \zeta_{k_{1}}^{N}+\frac{1}{2} \delta N\right)+\delta\left(\delta \zeta_{k_{1}}^{N}-\frac{1}{2} \delta N\right)\right] .
$$


Here we use $P(x)$ to denote the probability distribution function. This should not be confused with the power spectrum $P_{\zeta}$. The contribution from the entropy perturbation $\delta \zeta_{k_{1}}^{S}$ is still Gaussian.

Note that here we assumed for simplicity that the probability to pick up trajectory $A$ and $B$ are equal. This seems natural in our discussion because we are talking about a spontaneous breaking of an approximate symmetry. One can generalize the discussion straightforwardly to the case with unequal probability between trajectories. With unequal probability, we can also provide a theory for the CMB cold spot in the multi-stream inflation framework [22].

Note that the one point distribution function is not very easy to probe, especially for one particular value $k_{1}$. So we now proceed to discuss the other kind of non-Gaussianity produced by multi-stream inflation. The idea is that the amount of perturbation at comoving wave number $k_{1}$ and the amount of perturbation at comoving wave number $k_{1}<k<k_{2}$ are correlated. The correlation comes from the fact that the following three effects are $100 \%$ correlated with each other: 1. An isocurvature perturbation deciding whether to go along $A$ or $B$. 2. The difference between $P_{\zeta}^{A}$ and $P_{\zeta}^{B}$. 3. The difference of the e-folding number $\delta N$ between $A$ and $B$.

The three point correlation function can be written as

$$
\left\langle\zeta_{k_{1}} \zeta_{k} \zeta_{k}\right\rangle=\left\langle\zeta_{0} \zeta_{k} \zeta_{k}\right\rangle+\left\langle\delta \zeta_{k_{1}}^{S} \zeta_{k} \zeta_{k}\right\rangle+\left\langle\delta \zeta_{k_{1}}^{N} \zeta_{k} \zeta_{k}\right\rangle
$$

As we have neglected non-Gaussianities from sources other than the multi-stream effect, we have

$$
\left\langle\zeta_{0} \zeta_{k} \zeta_{k}\right\rangle=0
$$

The two terms from entropy modes and $\delta N$ can be written as an integration over the product of the three points and the probability distribution function,

$$
\left\langle\delta \zeta_{k_{1}}^{S, N} \zeta_{k} \zeta_{k}\right\rangle=\int_{-\infty}^{\infty} d \delta \zeta_{k_{1}}^{S, N} d \zeta_{k} P\left(\delta \zeta_{k_{1}}^{S, N}, \zeta_{k}\right) \delta \zeta_{k_{1}}^{S, N} \zeta_{k} \zeta_{k}
$$

with the combined probability distribution function of $\delta \zeta_{k_{1}}^{S, N}$ and $\zeta_{k}$,

$$
P\left(\delta \zeta_{k_{1}}^{S, N}, \zeta_{k}\right)=P\left(\delta \zeta_{k_{1}}^{S, N}\right)\left[\frac{e^{-\frac{\zeta_{k}^{2}}{2 \sigma_{A}^{2}}}}{\sqrt{2 \pi} \sigma_{A}} \theta\left(\delta \zeta_{k_{1}}^{S}\right)+\frac{e^{-\frac{\zeta_{k}^{2}}{2 \sigma_{B}^{2}}}}{\sqrt{2 \pi} \sigma_{B}} \theta\left(-\delta \zeta_{k_{1}}^{S}\right)\right]
$$


where $P\left(\delta \zeta_{k_{1}}^{S}\right)$ takes a form of Gaussian distribution $\frac{1}{\sqrt{2 \pi} \sigma_{1}^{S}} \exp \left\{-\frac{\left(\delta \zeta_{k_{1}}^{S}\right)^{2}}{2\left(\sigma_{1}^{S}\right)^{2}}\right\}$, and $P\left(\delta \zeta_{k_{1}}^{N}\right)$ is given in Eq. (8) with $\left(\sigma_{1}^{S}\right)^{2}=\left\langle\left(\zeta_{k_{1}}^{S}\right)^{2}\right\rangle, \sigma_{A}^{2}=\left\langle\zeta_{k}^{2}\right\rangle_{A}$ and $\sigma_{B}^{2}=\left\langle\zeta_{k}^{2}\right\rangle_{B} \cdot \theta(x)$ is the Heaviside step function. To avoid $\delta$ functions, we use the normalization that the fluctuation is averaged over one Hubble volume. (A familiar example is that in this normalization, the fluctuation of the inflaton takes the form $\delta \varphi \sim \frac{H}{2 \pi}$ ).

One can perform the integration in Eq. (11) and get

$$
\left\langle\zeta_{k_{1}} \zeta_{k} \zeta_{k}\right\rangle \simeq x P_{\zeta}^{1 / 2}\left(P_{\zeta}^{A}-P_{\zeta}^{B}\right)
$$

where $x \equiv \delta \zeta_{k_{1}} / \zeta_{k_{1}}$ denotes the fraction of extra fluctuation from the multi-stream effect.

Now we compare the above result with the local form non-Gaussianity to estimate $f_{N L}$ in the multi-stream model. The local form $f_{N L}$ is defined as

$$
\zeta=\zeta_{g}+\frac{3}{5} f_{N L}\left(\zeta_{g}^{2}-\left\langle\zeta_{g}^{2}\right\rangle\right)
$$

or we can rewrite the above equation as

$$
f_{N L} \simeq \frac{\left\langle\zeta^{3}\right\rangle}{\left\langle\zeta^{2}\right\rangle\left\langle\zeta^{2}\right\rangle}
$$

Comparing Eqs. (13) and (15), we have

$$
f_{N L} \simeq x P_{\zeta}^{-1 / 2}\left(\frac{P_{\zeta}^{A}-P_{\zeta}^{B}}{P_{\zeta}}\right)
$$

Note that $P_{\zeta}^{-1 / 2} \simeq 10^{5}$ is a large number. We can have $f_{N L} \simeq 100$ when $\left(P_{\zeta}^{A}-P_{\zeta}^{B}\right) / P_{\zeta}^{A} \simeq$ $3 \times 10^{-3}$ and $x \simeq 0.3$. As we shall show, this needs a tuning of the shape of the potential of order $1 \%$. For $f_{N L} \simeq 10$, the tuning is of order $10 \%$. Note that this non-Gaussianity appears only when one wave number is near $k_{1}$ ("near" denotes about one e-fold during inflation), $f_{N L}$ is strongly scale dependent, and should have large running.

We have not calculated the shape of non-Gaussianity for multi-stream inflation in the present paper. However, one note that non-Gaussianity is produced when $k \gg k_{1}$, known as the squeezed limit in the literature. So we expect that the shape of non-Gaussianity should be similar to the local shape, with large running. So the local form estimator $f_{N L}$ should apply for our model.

Finally, let us discuss the sign of $f_{N L}$. In multi-stream inflation, the sign of $f_{N L}$ is determined so that a positive $\delta \zeta_{k_{1}}$ results in a larger perturbation at scale $k_{1}<k<k_{2}$. 


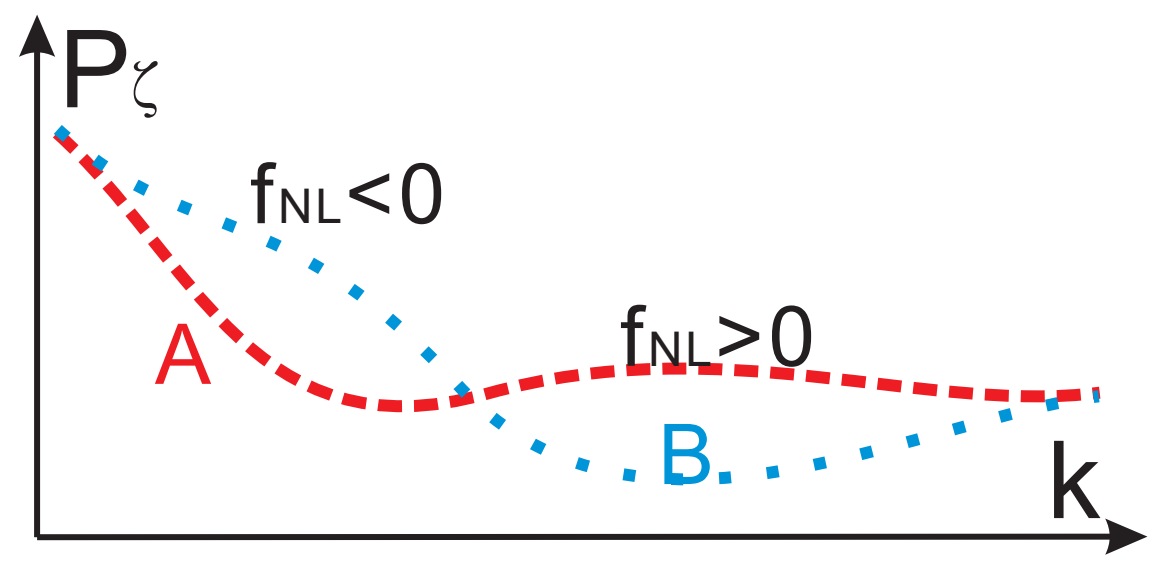

FIG. 2: We illustrate the scalar power spectrum as a function of scale $k$. The red (dashed) line denotes the trajectory $A$, and the blue (dotted) line denotes the trajectory $B$. If a perturbation $\delta \zeta_{k_{1}}>0$ leads to path $A$, then we have $f_{N L}<0$ on larger length scales and $f_{N L}>0$ on smaller length scales.

For example, when a fluctuation $\delta \zeta_{k_{1}}>0$ decides that the field roll to trajectory $A$, with $\zeta_{A}>\zeta_{B}$, then $f_{N L}>0$.

It is also interesting to note that in the multi-stream model, $f_{N L}$ can usually cross zero. It is because $P_{\zeta}^{A}$ may, say, larger than $P_{\zeta}^{B}$ on some scales, than become smaller than $P_{\zeta}^{B}$ on some other scales. As an example, when the deformation of the potential is "symmetric",

$$
\int_{\varphi_{1}}^{\varphi_{2}} \delta V d \varphi=0
$$

and the slow roll parameters satisfy $\mathcal{O}(\epsilon)>\mathcal{O}\left(\eta^{2}, \xi^{2}\right)$. In the lowest order of slow roll approximation, one have

$$
\int_{\varphi_{1}}^{\varphi_{2}} d \varphi\left(P_{\zeta}^{A}-P_{\zeta}^{B}\right)=0 .
$$

So in this example, $f_{N L}$ must change sign at a certain scale. This special kind of running of the non-Gaussianity is illustrated in Fig,2

\section{SUMMARY OF SIGNATURES FOR MULTI-STREAM INFLATION}

In this section, we will give a summary of signatures for multi-stream inflation. As we have mentioned in Section $2, \delta \zeta_{k_{1}}$ appears as an additional power at scale $k_{1}$ in the CMB. This additional power can not be arbitrarily large, because otherwise it should have been observed in the experiments. To be in accordance with CMB experiments, we require this 
anomaly power to satisfy $\delta \zeta_{k_{1}}<x_{m} P_{\zeta}^{1 / 2}$, where $x_{m}$ is determined by the error bar of CMB experiments, which varies when we choose $k_{1}$ to be different scales. Usually, one needs some fine tunning to satisfy the above constraint. In this section, we also discuss this tuning issue in more detail.

First of all, the amount of tuning depends on the detail of the potential, thus depends on the detail of string landscape. For example, if the trajectories $A$ and $B$ are completely symmetric, then there is no tuning at all. However, in this case, there are no new signatures for multi-stream inflation either.

To analyze the relation between tuning and signatures for multi-stream inflation further, let us calculate the relation between $\delta N$ and $\delta V$. We use the slow roll parameters

$$
\epsilon \equiv \frac{M_{p}^{2}}{2}\left(\frac{V^{\prime}}{V}\right)^{2}, \quad \eta \equiv M_{p}^{2} \frac{V^{\prime \prime}}{V} \quad \xi^{2} \equiv M_{P}^{4} \frac{V^{\prime} V^{\prime \prime \prime}}{V^{2}} .
$$

We first investigate the parameter regime where $\epsilon$ is not too small. To be more explicit, $\mathcal{O}(\epsilon)>\mathcal{O}\left(\eta^{2}, \xi^{2}\right)$, and with "symmetric" deformation of potential:

$$
\int_{\varphi_{1}}^{\varphi_{2}} \delta V d \varphi=0
$$

With these assumptions, $\delta N$ can be written as

$$
\delta N=\int_{\varphi_{1}}^{\varphi_{2}} d \varphi\left(\frac{V+\delta V}{M_{p}^{2}\left(V^{\prime}+\delta V^{\prime}\right)}-\frac{V}{M_{p}^{2} V^{\prime}}\right) \simeq \int_{\varphi_{1}}^{\varphi_{2}} d \varphi \frac{V}{M_{p}^{2} V^{\prime}}\left(\frac{\delta V}{V}-\frac{\delta V^{\prime}}{V^{\prime}}\right)
$$

The integration in Eq. (21) can be expanded in powers of the slow roll parameters. So the natural value of $\delta N$ is

$$
\delta N_{\text {natural }} \simeq\left(N_{1}-N_{2}\right)^{2}\left(-4 \eta+\frac{3 \eta^{2}}{\epsilon}-\frac{\xi^{2}}{\epsilon}\right) \frac{\delta V}{V} .
$$

We find that $\delta N$ is suppressed by slow roll parameters. Note that we have assumed $\int \delta V(\varphi-$ $\left.\varphi_{1}\right) d \varphi \simeq\left(\varphi_{2}-\varphi_{1}\right)^{2} \delta V$. If for some reasons we have $\int \delta V\left(\varphi-\varphi_{1}\right) d \varphi \ll\left(\varphi_{2}-\varphi_{1}\right)^{2} \delta V$, then $\delta N$ can be much smaller than given in Eq. (22).

To summarize the experimental signatures for multi-stream inflation, we propose two inequalities to divide the parameter space of multi-stream inflation into four regions.

As discussed at the beginning of this section, current CMB experiments do not allow large features. A condition $\delta \zeta_{k_{1}}<x_{m} P_{\zeta}^{1 / 2}$ is needed for multi-stream inflation to be consistent with CMB observations. If the above inequality is nearly saturated, then CMB experiments in the near future can detect the CMB features at scale $k_{1}$. 
Another inequality comes from the comparison of $\delta V / V$ and $P_{\zeta}^{1 / 2} / x$, where $x \equiv \delta \zeta_{k_{1}} / P_{\zeta}^{1 / 2}$. As shown in Eqs. (3) and (16), this comparison determines whether $f_{N L}$ is larger or smaller than $\mathcal{O}(1)$, thus detectable or not in the near future.

There is no estimator as sharp as $f_{N L}$ for the power asymmetry. So before explicitly fitting the multi-stream inflation model with CMB data, it is less clear how large power asymmetry can be detected in the next generation of experiments. However, one should note that the detection of $f_{N L}$ in Eq. (16) indicates a difference between $P_{\zeta}^{A}$ and $P_{\zeta}^{B}$. So here, we take the existence of large non-Gaussianity to be the condition for observable amount of power asymmetry. One should note that the detection of power asymmetry is in principle easier than the detection of $f_{N L}$, because the detection of power asymmetry does not need information for $\zeta_{k_{1}}$. We are glad to see whether improved methods can be proposed for detecting the power asymmetry.

Using the above two inequalities, the four parameter regions for multi-stream inflation are:

- When $\delta \zeta_{k_{1}} \ll x_{m} P_{\zeta}^{1 / 2}$ and $\delta V / V<P_{\zeta}^{1 / 2} / x$, there is no observable signature for multistream inflation. There may be an isocurvature perturbation, which is different from single field inflation. However, this isocurvature perturbation can also arise in simpler two field models.

- When $\delta \zeta_{k_{1}} \lesssim x_{m} P_{\zeta}^{1 / 2}$ and $\delta V / V<P_{\zeta}^{1 / 2} / x$, there is one signature for multi-stream inflation: a feature in the scalar power spectrum at scale $k_{1}$. Such features in the scalar power spectrum may be responsible for the features in the WMAP data.

- When $\delta \zeta_{k_{1}} \lesssim x_{m} P_{\zeta}^{1 / 2}$ and $\delta V / V>P_{\zeta}^{1 / 2} / x$, there are three signatures for multi-stream inflation: The first signature is as that in the above case: the feature in the spectrum at scale $k_{1}$. The second signature is that there will be an asymmetry in the powers between $k_{1}<k<k_{2}$ in one patch of the sky of scale $k_{1}$ and another. This could be responsible for the hemispherical power asymmetry. The third prediction is nonGaussianity. The non-Gaussianity is of order

$$
f_{N L} \simeq x P_{\zeta}^{-1 / 2} \frac{\delta V}{V}>1
$$

As discussed in the former section, $f_{N L}$ in our multi-stream model is largest in the squeezed limit because $k_{1} \ll k$, and $f_{N L}$ usually crosses zero. 
Now we estimate whether this case is natural or not. The natural value of $\delta N$ can be estimated using Eq. (22),

$$
\delta N_{\text {natural }} \simeq\left(N_{1}-N_{2}\right)^{2} \mathcal{O}(\epsilon) P_{\zeta}^{1 / 2} f_{N L} / x
$$

In order that perturbation on the scale $k_{1}$ is not ruled out by experiments, we need $\delta \zeta_{k_{1}}<x_{m} P_{\zeta}^{1 / 2}$. The amount of tuning for this parameter region is measured by

$$
\frac{\delta \zeta_{k_{1}}}{\delta N_{\text {natural }}} \simeq \frac{\delta N}{\delta N_{\text {natural }}} \simeq \frac{x_{m}^{2}}{\left(N_{1}-N_{2}\right)^{2} \mathcal{O}(\epsilon) f_{N L}}
$$

For example, if we have multi-stream inflation for 3 e-folds, $\epsilon=\mathcal{O}(0.01)$, and $x_{m}^{2}=0.1$, we have $\delta \zeta_{k_{1}} / \delta N_{\text {natural }} \simeq 1 / f_{N L}$. To be more explicit, there is one potential in 10 potentials that can produce $f_{N L}=10$, and one in 100 that can produce $f_{N L}=100$. Assume that future experiments show $f_{N L}=10$, then 3 features in the scalar power spectrum can naturally achieve that.

- When $\delta \zeta_{k_{1}} \ll x_{m} P_{\zeta}^{1 / 2}$ and $\delta V / V>P_{\zeta}^{1 / 2} / x$, there is large non-Gaussianity, asymmetry on powers in different patches of the sky, and no feature in the power spectrum. However, more fine tuning is needed for this possibility than the former one.

There is potentially one more signature of multi-stream inflation: the correlation between the curvature perturbation and the isocurvature perturbation. However, as we need to go to detailed models to calculate this correlation, we shall not consider it in the present work. We hope to address this issue in the future.

If $\epsilon$ is even smaller than $\eta^{2}$ and $\chi^{2}$, then we are unable to perform the expansion (22) in the slow roll parameters. In this case, $\delta N / N \sim \delta V / V$, and we need more fine tuning to get the desired non-Gaussianity and anisotropy for perturbation powers for multi-stream inflation.

\section{CONCLUSION}

To conclude, we have described the multi-stream inflation model, and calculated the density perturbation and non-Gaussianity of the model.

We find that the model can be parameterized by the $\delta V / V$ and $\delta N$. We show that signatures such as non-Gaussianity, features in the CMB and the hemispherical power asymmetry can be produced in corresponding parameter regions. 
We also note that isocurvature perturbation at $\varphi_{1}$ and $\varphi_{2}$ may produce interesting physics. We hope we can address these issues in some future work. We also hope to build more explicit models of multi-stream inflation, and investigate the signatures of multi-stream inflation in more detail in the future. Finally, the perturbations are studied semi-quantitatively in this paper. It is important to calculate the perturbations more precisely and determine the $\mathcal{O}(1)$ coefficients. To do so, one needs to investigate the cosmic perturbation theory for a quantum state with two decohered branches. We also leave this calculation to future work.

\section{Acknowledgments}

We thank Bruce Bassett, Robert Brandenberger, Yifu Cai, Xingang Chen, Qing-Guo Huang and Xian Gao for discussion. This work was supported by NSFC Grant No. 10525060, and a 973 project grant No. 2007CB815401.

[1] R. Bousso and J. Polchinski, JHEP 0006, 006 (2000) arXiv:hep-th/0004134]. S. B. Giddings, S. Kachru and J. Polchinski, Phys. Rev. D 66, 106006 (2002) arXiv:hep-th/0105097. S. Kachru, R. Kallosh, A. Linde and S. P. Trivedi, Phys. Rev. D 68, 046005 (2003) arXiv:hep-th/0301240]. M. R. Douglas, JHEP 0305, 046 (2003) arXiv:hep-th/0303194.

[2] Q. G. Huang and S. H. Tye, arXiv:0803.0663 [hep-th].

[3] E. Komatsu et al. [WMAP Collaboration], Astrophys. J. Suppl. 180, 330 (2009) arXiv:0803.0547 [astro-ph]].

[4] T. J. Allen, B. Grinstein and M. B. Wise, Phys. Lett. B 197, 66 (1987).

[5] H. M. Hodges, G. R. Blumenthal, L. A. Kofman and J. R. Primack, Nucl. Phys. B 335, 197 (1990).

[6] D. S. Salopek and J. R. Bond, Phys. Rev. D 42, 3936 (1990).

[7] T. Falk, R. Rangarajan and M. Srednicki, Astrophys. J. 403, L1 (1993) arXiv:astro-ph/9208001].

[8] A. Gangui, F. Lucchin, S. Matarrese and S. Mollerach, Astrophys. J. 430, 447 (1994) arXiv:astro-ph/9312033.

[9] E. Komatsu and D. N. Spergel, arXiv:astro-ph/0012197; 
E. Komatsu and D. N. Spergel, Phys. Rev. D 63, 063002 (2001), arXiv:astro-ph/0005036;

[10] V. Acquaviva, N. Bartolo, S. Matarrese and A. Riotto, Nucl. Phys. B 667, 119 (2003) arXiv:astro-ph/0209156.

[11] A. D. Linde and V. F. Mukhanov, Phys. Rev. D 56, 535 (1997) arXiv:astro-ph/9610219.

[12] M. Alishahiha, E. Silverstein and D. Tong, Phys. Rev. D 70, 123505 (2004) arXiv:hep-th/0404084.

[13] J. M. Maldacena, JHEP 0305, 013 (2003) arXiv:astro-ph/0210603.

[14] D. Babich, P. Creminelli and M. Zaldarriaga, JCAP 0408, $009 \quad$ (2004) [arXiv:astro-ph/0405356]; JCAP 0701, 002 (2007) arXiv:hep-th/0605045].

[15] N. Bartolo, E. Komatsu, S. Matarrese and A. Riotto, Phys. Rept. 402, 103 (2004) arXiv:astro-ph/0406398].

[16] X. Chen, R. Easther and E. A. Lim, JCAP 0706, 023 (2007) arXiv:astro-ph/0611645]. K. Fang, B. Chen and W. Xue, Phys. Rev. D 77, 063523 (2008) arXiv:0707.1970 [astroph]]. M. Li, T. Wang and Y. Wang, JCAP 0803, 028 (2008) arXiv:0801.0040 [astro-ph]]. Q. G. Huang, Phys. Lett. B 669, 260 (2008) arXiv:0801.0467 [hep-th]]. X. Chen, R. Easther and E. A. Lim, JCAP 0804, 010 (2008) arXiv:0801.3295 [astro-ph]]. S. W. Li and W. Xue, arXiv:0804.0574 [astro-ph]. X. Gao, JCAP 0806, 029 (2008) arXiv:0804.1055 [astroph]]. M. Li, C. Lin, T. Wang and Y. Wang, arXiv:0805.1299 [astro-ph]. S. Li, Y. F. Cai and Y. S. Piao, Phys. Lett. B 671, 423 (2009) arXiv:0806.2363 [hep-ph]]. W. Xue and B. Chen, arXiv:0806.4109 [hep-th]. Q. G. Huang, Phys. Rev. D 78, 043515 (2008) arXiv:0807.0050 [hep-th]]. Q. G. Huang, JCAP 0809, 017 (2008) arXiv:0807.1567 [hep-th]]. Y. S. Piao, arXiv:0807.3813 [gr-qc]. Q. G. Huang and Y. Wang, JCAP 0809, 025 (2008) arXiv:0808.1168 [hep-th]]. Q. G. Huang, JCAP 0811, 005 (2008) arXiv:0808.1793 [hep-th]]. Y. Ling and J. P. Wu, arXiv:0809.3398 [hep-th]. B. Chen, Mod. Phys. Lett. A 23, 1577 (2008). P. Chingangbam and Q. G. Huang, arXiv:0902.2619 [astro-ph.CO]. Y. F. Cai, W. Xue, R. Brandenberger and X. Zhang, arXiv:0903.0631 [astro-ph.CO]. Q. G. Huang, arXiv:0903.1542 [hep-th]. X. Gao, B. Hu, arXiv:0903.1920 [astro-ph.CO].

[17] Aalok Misra, Pramod Shukla, Nucl.Phys.B810:174-192,2009, e-Print: arXiv:0807.0996 [hepth], Aalok Misra, Pramod Shukla, Nucl.Phys.B800:384-400,2008, arXiv:0712.1260 [hep-th]. I. Zaballa, Y. Rodriguez, and D. H. Lyth: arXiv:astro-ph/0603534 (JCAP 0606 (2006) 013). D. H. Lyth and Y. Rodriguez: arXiv:astro-ph/0502578 (Phys. Rev. D71 (2005) 123508). D. H. 
Lyth and Y. Rodriguez: arXiv:astro-ph/0504045 (Phys. Rev. Lett. 95 (2005) 121302)

[18] M. Li and Y. Wang, JCAP 0809, 018 (2008) [arXiv:0807.3058 [hep-th]].

[19] L. Covi, J. Hamann, A. Melchiorri, A. Slosar and I. Sorbera, Phys. Rev. D 74, 083509 (2006) arXiv:astro-ph/0606452].

[20] H. K. Eriksen, F. K. Hansen, A. J. Banday, K. M. Gorski and P. B. Lilje, Astrophys. J. 605, 14 (2004) [Erratum-ibid. 609, 1198 (2004)] [arXiv:astro-ph/0307507]. A. L. Erickcek, M. Kamionkowski and S. M. Carroll, Phys. Rev. D 78, 123520 (2008) [arXiv:0806.0377] [astro$\mathrm{ph}]$.

[21] A. A. Starobinsky, JETP Lett. 42, 152 (1985), M. Sasaki and E. D. Stewart, Prog. Theor. Phys. 95, 71 (1996) arXiv:astro-ph/9507001]. D. H. Lyth, K. A. Malik and M. Sasaki, JCAP 0505, 004 (2005) arXiv:astro-ph/0411220.

[22] Niayesh Afshordi, Anže Slosar, Yi Wang, in preparation. 